\title{
Función sexual en mujeres usuarias de terapia de reemplazo hormonal
}

\author{
Juan E Blumel M, Francisca Bravo $M^{a}$, \\ Marcia Recavarren Aa, Salvador Sarrá C. \\ Sexual function in postmenopausal \\ women using hormone replacement \\ therapy
}

Background: The loss of sexuality observed in the climacteric period is difficult to evaluate. An important advance has been the development of the Female Sexual Function Index (FSFI), a test based on the norms of the International Consensus Development Conference on Sexual Female Dysfunctions. Aim: To study the effects of hormone replacement therapy (HRT) on sexuality, applying the FSFI. Material and methods: The FSFI was applied to 300 healthy women between 45-64 years, sexually active, beneficiaries of the Southern Metropolitan Health Service. Results: The mean age of the sample studied was $51 \pm 5$ years, $27 \%$ were HRT users, $21 \%$ had had an hysterectomy and $98 \%$ had a stable couple. The total score of the FSFI decreased from $27.3 \pm 5.8$ in women between 45 and 49 years of age to $19.3 \pm 7.0$ in women between 60 and 64 years ( $\mathrm{p}<0.01$ ). A significantly better sexuality was observed in HRT users, with FSFI scores of $28.1 \pm 5.5$ and $24.6 \pm 6.8$ in HRT users and non users, respectively ( $p$ $<0.01$ ). Women on HRT obtained a higher score in all of the test domains, especially in lubrication, orgasm and sexual satisfaction. Conclusions: Female sexuality decreases with aging. HRT users have a better sexual function than non users (Rev Méd Chile 2003; 131: 1251-55 ).

(Key Words: Estrogen replacement therapy; Menopause; Postmenopause)

Recibido el 20 de enero, 2003. Aceptado en versión corregida el 2 de septiembre, 2003. Departamento Medicina Sur. Facultad de Medicina, Universidad de Chile. Hospital Barros Luco-Trudeau.

aAlumna de Medicina, Universidad de Chile.

E climaterio produce no sólo declinación de os niveles plasmáticos de estrógeno, sino también cambios orgánicos y psicológicos que afectan la calidad de vida de la mujer de edad media ${ }^{1}$. Dentro del concepto de calidad de vida, la sexualidad ocupa un lugar importante ${ }^{2}$, razón por la cual el deterioro que se observa con la edad y el climaterio es un proceso relevante ${ }^{3,4}$.

Correspondencia a: María Francisca Bravo M. Simón González 7801 casa O, La Reina, Santiago de Chile.

Teléfono: 09-1003180. E mail: Panchitab_@hotmail.com.
Es difícil estudiar un fenómeno tan complejo como la sexualidad humana, y si a este hecho le sumamos los problemas metodológicos que tienen la mayoría de los estudios que la investigan, entenderemos las dificultades que se presentan al interpretar y comparar los resultados publicados sobre esta área de la conducta humana ${ }^{5}$. Rosen, basado en el International Consensus Development Conference on Female Sexual Dysfunctions ${ }^{6}$, reunió a un grupo de expertos y desarrolló un instrumento para evaluar la sexualidad femenina, 
que denominó «ndice de Función Sexual Femenina» (IFSF) ${ }^{7}$.

En una publicación reciente observamos que mujeres en etapa postmenopáusica creen que el climaterio deteriora su salud y que la utilización de la terapia de reemplazo hormonal (TRH) las hace sentir más saludables ${ }^{8}$. Un estudio previo mostró que las usuarias de TRH presentaban una disminución del riesgo de disfunción sexual ${ }^{1}$. Como recientemente hemos validado en nuestro medio el «ndice de Función Sexual Femenina»de Rosen ${ }^{9}$, nos interesó aplicar este instrumento en usuarias de TRH para conocer en mayor profundidad los efectos de esta terapia en la sexualidad.

\section{Pacientes y MÉTodo}

Pacientes. La muestra fue tomada de un universo estimado en 58.000 mujeres de 45 a 64 años de edad, beneficiarias del Servicio de Salud Metropolitano Sur de Santiago. Utilizando el programa estadístico Epiinfo 6.04 para el cálculo del tamaño muestral, con una frecuencia esperada de trastornos de sexualidad de $50 \%^{3}$, un error máximo aceptable de $5 \%$ y con $95 \%$ de confianza, obtuvimos que el tamaño mínimo de la muestra debiera ser de 382 personas. Como sabemos que el porcentaje de mujeres sexualmente activas, grupo que nos interesa para el presente estudio, es $78,6 \%^{3}$, el número final a estudiar es de 300 mujeres. Las encuestadoras fueron dos estudiantes de sexto año de medicina de sexo femenino. Cada encuestada procedió a leer y contestar en forma personal las preguntas. La entrevista se realizó privadamente en el CDT del Hospital Barros Luco. Las estudiantes de medicina procedieron a invitar a participar en el estudio a 360 mujeres que concurrieron como acompañantes de pacientes ambulatorios que consultaban en el Centro de Diagnóstico y Tratamiento del Hospital Barros Luco-Trudeau en el mes de diciembre de 2002. Sesenta mujeres $(16,7 \%)$ rechazaron participar en esta investigación, quedando el grupo de estudio constituido por 300 encuestadas. Criterios de inclusión: mujeres con actividad sexual en los últimos tres meses y cuyo estado de salud fuera normal, definido por el National Center for Health Statistics como aquella condición compatible con el desempeño de las actividades rutinarias ${ }^{10}$. Los criterios de exclusión fueron embarazos, patologías psiquiátricas o déficit mental que pudiera dificultar la interpretación de las preguntas.

Diseño. El diseño de esta investigación corresponde a un estudio de corte transversal en el cual se evalúa la sexualidad femenina mediante la encuesta «ndice de Función Sexual Femenina». Este cuestionario ha sido validado al castellano en un estudio previo ${ }^{9}$. El cuestionario consiste en 19 preguntas agrupadas en seis dominios: deseo, excitación, lubricación, orgasmo, satisfacción y dolor. Cada pregunta tiene 5 ó 6 alternativas de respuesta, con un puntaje que varía desde 0 a 5 en cada pregunta. El puntaje total obtenido en la encuesta se obtiene mediante la suma aritmética de los productos obtenidos al multiplicar el resultado de cada pregunta por un factor. A mayor puntaje mejor sexualidad. Además, se consignó la edad, uso de TRH, presencia de pareja estable e histerectomía. Se consideró usuaria de TRH a aquella mujer que se encontraba en el momento de la encuesta usando reemplazo hormonal; aquellas que no lo hacían, se definieron como nousuarias.

Análisis estadístico. El análisis de los datos se realizó con el programa Epi-Info versión 6.04 (Centers for Disease Control, USA; OMS, Suiza). La comparación entre los grupos fue evaluada con ANOVA o con Kruskall-Wallis, según homogeneidad de la varianza medida con la prueba de Bartlet. Las diferencias en porcentajes se evaluaron con chi cuadrado. Los resultados se expresan como promedio \pm desviación estándar, considerándose significación valores de $\mathrm{p}<0,05$.

\section{RESULTADOS}

Se solicitó a 360 mujeres que contestaran la encuesta «ndice de Función Sexual Femenina», rechazando $60(16,7 \%)$ a participar en ella. Por lo tanto, se presentan los resultados de trescientas mujeres. En la Tabla 1 se muestra la distribución etárea de la población estudiada; la edad media fue $51,1 \pm 5,2$, con un rango de 45 a 64 años. En este grupo, 81 mujeres (27\%) utilizaba terapia de reemplazo hormonal y $64(21,3 \%)$, estaba histerectomizada; porcentaje que aumenta desde 14,8\% 
Tabla 1. C aracterísticas de la población estudiada: distribución etárea, frecuencia de histerectomía y uso de T RH

\begin{tabular}{|c|c|c|c|c|c|c|}
\hline \multirow[t]{2}{*}{ Edad } & \multirow[b]{2}{*}{$\mathrm{n}$} & \multirow[b]{2}{*}{$\%$} & \multicolumn{2}{|c|}{ TRH } & \multicolumn{2}{|c|}{ Histerectomizadas } \\
\hline & & & $\mathrm{n}$ & $\%$ & $n$ & $\%$ \\
\hline $45-49$ & 135 & 45,0 & 30 & 22,2 & 20 & 14,8 \\
\hline $50-54$ & 96 & 32,0 & 32 & 33,3 & 20 & 20,8 \\
\hline $55-59$ & 40 & 13,3 & 12 & 30,0 & 14 & 35,0 \\
\hline $60-64$ & 29 & 9,7 & 7 & 24,1 & 10 & 34,5 \\
\hline Total & 300 & 100,0 & 81 & 27,0 & 64 & 21,3 \\
\hline
\end{tabular}

en el segmento etáreo de $45-49$ años a $34,5 \%$ a los 60-64 años ( $p<0,01)$. Todas, de acuerdo a los criterios de inclusión habían tenido actividad sexual en los últimos tres meses; teniendo $98 \%$ de ellas pareja estable.

En la Tabla 2 se observa que los promedios del puntaje total del Indice de Función Sexual Femenina disminuye significativamente con la edad. Así por ejemplo, el puntaje del grupo etáreo entre 4549 años fue de $27,3 \pm 5,8$ y cayó a 19,3 37,0 en el grupo entre 60-64 años ( $\mathrm{p}<0,0001)$.

Los puntajes de la prueba IFSF y algunas características clínicas de las mujeres con y sin TRH se aprecian en la Tabla 3. No se encontró diferencias en el promedio de edad entre las usuarias y no-usuarias de terapia hormonal (51, $6 \pm 4,9$ vs $50,9 \pm 5,4$ respectivamente), pero sí, en el porcentaje de histerectomizadas; así, mientras a $29,6 \%$ de la usuarias se les había realizado histerectomía, a $18,3 \%$ de las no-usuarias se les había practicado dicha operación $(p<0,04)$. Con respecto a los resultados obtenidos en el IFSF, se

Tabla 2. Puntaje total del Indice de Función Sexual Femenina según edad

\begin{tabular}{|ccc|}
\hline Edad & Promedio \pm DE & $n$ \\
\hline $45-49$ & $27,3 \pm 5,8$ & 135 \\
$50-54$ & $25,5 \pm 6,2$ & 96 \\
$55-59$ & $24,1 \pm 7,4$ & 40 \\
$60-64$ & $19,3 \pm 7,0$ & 29 \\
\hline
\end{tabular}

Anova $\mathrm{p}<0,0001$

Tabla 3. Características clínicas y puntaje del Indice de Función Sexual Femenina en usuarias y no-usuarias de T RH

\begin{tabular}{|c|c|c|c|c|}
\hline & & TRH (+) & TRH (-) & $\mathrm{p}$ \\
\hline & $\mathrm{n}$ & 81 (27\%) & 219 (73\%) & - \\
\hline & Edad & $51,6 \pm 4,9$ & $50,9 \pm 5,4$ & ns \\
\hline & Histerectomía & $24(29,6 \%)$ & $40(18,3 \%)$ & $<0,04$ \\
\hline శే శ & Deseo & $3,6 \pm 1,2$ & $3,2 \pm 1,3$ & $<0,04$ \\
\hline 寻 & Dolor & $5,2 \pm 1,0$ & $4,7 \pm 1,3$ & $<0,004$ \\
\hline छ & Orgasmo & $4,8 \pm 1,2$ & $4,1 \pm 1,4$ & $<0,0002$ \\
\hline (1) & Satisfacción & $5,1 \pm 1,2$ & $4,4 \pm 1,5$ & $<0,0004$ \\
\hline 0 & Excitación & $4,3 \pm 1,2$ & $3,7 \pm 1,3$ & $<0,0008$ \\
\hline : & Lubricación & $5,1 \pm 1,1$ & $4,4 \pm 1,5$ & $<0,00008$ \\
\hline \& & Puntaje total & $28,1 \pm 5,5$ & $24,6 \pm 6,8$ & $<0,00005$ \\
\hline
\end{tabular}


observa en todos los dominios de la prueba un puntaje significativamente mayor en usuarias de TRH. Las mayores diferencias a favor de la TRH se observan en la lubricación vaginal $(5,1 \pm 1,1$ vs $4,4 \pm 1,5 ; \mathrm{p}<0,00008)$, orgasmo $(4,8 \pm 1,2$ vs $4,1 \pm 1,4$; $\mathrm{p}<0,0002)$ y satisfacción $(5,1 \pm 1,2$ vs $4,4 \pm 1,5 ; \mathrm{p}$ $<0,0004)$. El puntaje total obtenido en mujeres con TRH fue $28,1 \pm 5,5$ y en mujeres sin TRH fue $24,6 \pm 6,8$ ( $p<0,00005)$.

\section{Discusión}

El descenso de los niveles circulantes de estrógenos que ocurre durante el climaterio disminuye la lubricación vaginal, afecta la mecánica del piso pélvico, cambia la configuración corporal y aumenta los trastornos del ánimo; traduciéndose todos estos efectos en una pérdida del deseo sexual ${ }^{11}$. Por esto, no es raro que la menopausia sea uno de los factores de riesgo más importante de disfunción sexual ${ }^{12}$.

La TRH revierte los trastornos climatéricos que deterioran la sexualidad. Sarrel ha mostrado que las usuarias de TRH tienen mejor lubricación ${ }^{13}$. Genazzani y Hildith señalan que la TRH mejora la sexualidad y la calidad de vida en general ${ }^{14,15}$. En un estudio previo, habíamos señalado que la TRH es un fuerte factor protector de riesgo de disfunción sexual ${ }^{3}$. Sin embargo, ninguno de estos estudios cumple con las normas del International Consensus Development Conference on Female Sexual Dysfunctions ${ }^{7}$.

Nosotros encontramos en este estudio que un porcentaje importante de mujeres utilizan terapia hormonal y que este hecho se mantiene incluso en los segmentos etáreos mayores. La función sexual, evaluada con un instrumento con propiedades psicométricas como el IFSF, mejora significativamente en las usuarias de TRH. El análisis de los resultados de los diferentes dominios de la prueba, así como el puntaje total obtenido, sugie-

\section{REFERENCIAS}

1. Blumel JE, Castelo-Branco C, Binfa L, Gramegna G, Tacla X, Aracena B et al. Quality of life after the menopause: a population study. Maturitas 2000; 34: 17-23. re que la terapia de remplazo hormonal es un factor protector importante de la función sexual femenina y que contribuye, mejorando la sexualidad, mediante la disminución del dolor, la mejoría del deseo, de la excitación, de la lubricación, de la satisfacción y del orgasmo.

Dentro de los sesgos a considerar, la edad es un factor determinante en la interpretación de los resultados de los diferentes estudios, debido al efecto negativo que tiene el envejecimiento sobre la función sexual ${ }^{16}$. En nuestro estudio no hay diferencia de edad entre las usuarias y no-usuarias de TRH, por lo que la mejor sexualidad observada en las usuarias no puede ser atribuida a este factor y elimina este sesgo de nuestros resultados. Otro aspecto importante, en la función sexual es la histerectomía, cirugía que aumenta significativamente el riesgo de disfunción sexual ${ }^{6,17}$. En este estudio encontramos que las usuarias de TRH tienen mayor prevalencia de histerectomía, situación que aminora el efecto positivo en la función sexual de la TRH. Por este motivo, es probable que la mejoría asociada al uso de TRH sea aún mayor que la descrita por nosotros.

Se debe tener presente que la función sexual no sólo depende de los niveles estrogénicos, sino que es una complementariedad de éstos con aspectos sicológicos, sociales, ambientales y culturales; factores que son propios de cada mujer y que le dan la característica de ser una cualidad individual ${ }^{14,18}$. Los médicos no siempre podemos actuar en los ámbitos socioculturales de la sexualidad, pero sí podemos modificar mediante la TRH aspectos biológicos de la función sexual femenina.

En conclusión, este estudio muestra que la TRH tiene una influencia positiva en la función sexual en mujeres entre 45-64 años de edad. No sólo mejora la lubricación y la dispareunia, sino que también tiene un efecto positivo sobre el orgasmo, la satisfacción, la excitación y el deseo sexual. Por lo tanto, su uso podría ser una alternativa para mujeres con disfunción sexual.
2. Hilditch JR, Lewis J, Peter A, Van Maris B, Ross A, FRANSSEN E ET AL. A menopause specific quality of life questionaire, development and psychometric properties. Maturitas 1996; 24: 161-75.

3. Blumel J, Araya H, Riquelme R, Castro G, Sánchez F, Gramegna G. Prevalencia de los trastornos de la 
sexualidad en mujeres climatéricas. Influencia de la menopausia y de la terapia de reemplazo hormonal. Rev Méd Chile 2002; 130: 1131-8.

4. Avis NE, Stelato R, Crawford S, Johannes C, LONGCOPE C. Is there an association between menopause status and sexual functioning? Menopause 2000; 7: 286-8.

5. Mc Coy NL. Methodological problems in the study of sexuality and the menopause. Maturitas 1998; 29: 51-60.

6. Basson R, Berman J, Burnett A, Derogatis L, FERGURSON D, Fourcroy J et al. Report of the International Consensus Development Conference on Female Sexual Dysfunction: definitions and classifications. J Urol 2000; 163: 888-93.

7. Rosen R, Brown C, Heiman J, Leiblum S, Meston C, Shabsigh R et al. The Female Sexual Function Index (FSFI): A Multidimensional Self-Report Instrument for the Assessment of Female Sexual Function. Journal of Sex \& Marital Therapy 2000; 26: 191-208.

8. Blumel J, Castelo-Branco C, Kerrigan N, Cancelo M, Blumel B, Haya J et al. Influences of hormone replacement therapy on postmenopausal women's health perceptions. Menopause 2003; 10: 235-40.

9. Blumel Je, Binfa L, Cataldo P, Carrasco A, Izaguirre $\mathrm{H}$, SARRA S. Indice de Función Sexual Femenina: una prueba para evaluar la sexualidad de la mujer. Rev Chil Obstet Ginecol. En prensa.

10. BRETT KM, Chong Y. Hormone Replacement Therapy: Knowledge and use in the United States. Hyattsville, Maryland: National Center for Health Statistics, 2001.
11. BaChmann GA. Influence of menopause on sexuality. IntJ Fertil Menopausal Study 1995; 40 Suppl 1: 16-22.

12. Mansfield PK, Косh PB, Voda AM. Midlife women's attributions for their sexual response changes. Health Care Women Int 2000; 21: 543-59.

13. SARREL PM. Effects of hormone replacement therapy on sexual psychophysiology and behavior in postmenopause. J Women's Health Gend Based Med 2000; 9 Suppl 1: S25-32.

14. Genazzani AR, Nicolucci A, Campagnoli C, CrosigNANI P, NAPPI C, SERRA GB ET al. Assessment of the QoL in Italian menopausal women: comparison between HRT users and non-users. Maturitas 2002; 42: 267-80.

15. Hibdich JR, Lewis J, Ross AH, Peter A, Van Maris B, FransSen E ET AL. A comparison of the effects of oral conjugated equine estrogen and transdermal estradiol-17 beta combined with an oral progestin on quality of life in postmenopausal women. Maturitas 1996; 24: 177-84.

16. KINGSBERG SA. The impact of aging on sexual function in women and their partners. Arch Sex Behav 2002; 31: 431-7.

17. Mazer NA, Leiblum SR, Rosen RC. The brief index of sexual functioning for women (BISF-W): a new scoring algorithm and comparison of normative and surgically menopausal populations. Menopause 2000; 7: 350-63.

18. Gramegna G, Blumel JE, Roncagliolo ME, Aracena B, Tacla X. Patrones de conducta sexual en mujeres chilenas. Rev Méd Chile 1998; 126: 162-8. 